\title{
Optimal Design of Bus Stop Locations Integrating Continuum Approximation and Discrete Models
}

\author{
Xiaoling Luo, ${ }^{1,2}$ Wenbo Fan ${ }^{D},{ }^{1}$ Yangsheng Jiang, ${ }^{1}$ and Jun Zhang ${ }^{3}$ \\ ${ }^{1}$ School of Transportation and Logistics, Southwest Jiaotong University, Chengdu, Sichuan 611756, China \\ ${ }^{2}$ Chongqing Jiaotong University, Chongqing Key Laboratory of Traffic \& Transportation, Chongqing 400074, China \\ ${ }^{3}$ Office of Science Research \& Development, Southwest Jiaotong University, Chengdu, Sichuan 611756, China
}

Correspondence should be addressed to Wenbo Fan; wbfan@swjtu.edu.cn

Received 3 June 2020; Revised 19 August 2020; Accepted 20 August 2020; Published 7 September 2020

Academic Editor: Kun Wang

Copyright ( $\odot 2020$ Xiaoling Luo et al. This is an open access article distributed under the Creative Commons Attribution License, which permits unrestricted use, distribution, and reproduction in any medium, provided the original work is properly cited.

\begin{abstract}
Although transit stop location problem has been extensively studied, the two main categories of modeling methodologies, i.e., discrete models and continuum approximation (CA) ones, seem have little intersection. Both have strengths and weaknesses, respectively. This study intends to integrate them by taking the advantage of CA models' parsimonious property and discrete models' fine consideration of practical conditions. In doing so, we first employ the state-of-the-art CA models to yield the optimal design, which serves as the input to the next discrete model. Then, the stop location problem is formulated into a multivariable nonlinear minimization problem with a given number of stop location variables and location constraint. The interior-point algorithm is presented to find the optimal design that is ready for implementation. In numerical studies, the proposed model is applied to a variety of scenarios with respect to demand levels, spatial heterogeneity, and route length. The results demonstrate the consistent advantage of the proposed model in all scenarios as against its counterparts, i.e., two existing recipes that convert CA model-based solution into real design of stop locations. Lastly, a case study is presented using real data and practical constraints for the adjustment of a bus route in Chengdu (China). System cost saving of $15.79 \%$ is observed by before-and-after comparison.
\end{abstract}

\section{Introduction}

Transit route design problem can be divided into two categories: transit network design and single transit route design [1-5]. Well-designed transit routes constitute as the basic bricks to the big transit network in many cities for defending the wide spread of roadway traffic congestion. The design of a single transit route mainly concerns the locations of stops/stations and the service headways/frequencies during the operation periods. Being physically inflexible (at least for a short term), transit stop locations affect the service accessibility to potential patrons as well as their experienced level of service in terms of, e.g., commercial speed. On the supply side, the design of stop locations also influences transit agency's operation efficiency in terms of vehicle fuel cost and vehicle fleet size, for instance.

The transit stop location problem has been extensively studied in the literature. Methodologically, two categories can be identified: discrete models and continuum approximation (CA) models. A majority of studies belong to the discrete-method category. For instance, Vuchic and Newell and Vuchic $[6,7]$ may be the two pioneering works. They sought to find the optimal interstation spacings of a rapid transit corridor to minimize passenger travel time and maximize number of passengers, respectively. Later on, Gleason [8] developed a set covering approach for locating bus stops. This work was extended by Murray with a hybrid set covering model, which determined the stop locations of an existing route segment as well as the locations of new stops for route extension in unserved areas [9]. Furth and Rahbee [10] optimized bus stop locations from a set of prespecified candidate stops in a bus route of Boston. Similarly, Chien and Qin [11] identified a set of demand points as candidate locations and proposed to minimize the total system cost through finding the optimal number and locations of bus stops. Recently, Ceder et al. [12] integrated 
the impacts of uneven topography into a bus stop location model to more precisely account for users' walking-access speed and vehicles' acceleration performance.

In the second category, CA models had been developed as an alternative option in locating transit stops. Instead of based on dozens of location variables, these models were built upon a single stop density/spacing variable or function. This parsimonious property endows the CA model with the high-efficient finding of the global optimum solution, or sometimes the closed-form solution. The first endeavor in this vein was made by Newell $[13,14]$. Later, Wirasinghe and Ghoneim [15] proposed a more general CA-based model for determining bus stop spacing (expressed as a function of location). Hurdle and Wirasinghe and Wirasinghe and Seneviratne $[16,17]$ analyzed the influence of stop spacing and line length with the objective function of system cost minimization. Medina et al. [18] applied a similar CA-based model to locate bus stops considering multiperiod demand in Santiago, Chile. Mostly recently, Su et al. [19] incorporated environmental factors into CA models for an e-bus stop location problem.

The CA models, however, have been criticized being too idealized with unrealistic assumptions, such as a continuous space for locating stops at anywhere along the route. Thus, it is recognized that the designs offered by CA models are not ready for implementation. Endeavors had been made to enhance the applicability of CA models. In Wirasinghe and Ghoneim and Medina et al. [15, 18], the continuous stop density/spacing function was discretized into specific locations via the integral method. Yet their models still lack the consideration of realistic street layout and practical location restrictions, e.g., intersections, bridges, and natural obstacles, where no bus stops should be placed.

This paper intends to fill the gap. We propose an optimization framework that integrates CA models with discrete ones for locating bus stops with respect to location constraint. The idealized design of the CA model serves as input to the discrete model, which accordingly defines a given number of stop location variables and formulates the location constraint. The corresponding problem is a nonlinear multivariate optimization problem. A heuristic solution algorithm is presented to find the optimal solution. To the best of our knowledge, this is the first work connecting $\mathrm{CA}$ and discrete models so as to furnish implementationready transit route designs.

The remainder of the paper is organized as follows. The next section introduces the existing CA and discretization models. After that, a novel optimization model is proposed for locating bus stops. In Section 3, the solution method is developed to solve the bus stop location problem model. Section 4 presents numerical studies of various experiments in a hypothetical route and a case study in Chengdu (China). Conclusions are drawn in the final section.

\section{Models}

Section 2.1 presents the state-of-the-art CA model of bus route design, followed by the existing recipes that discretize the solutions of the CA model into real designs. Sections 2 and 3 propose our discretization recipe that offers the improved designs and admit practical constraints on stop locations. Table 1 summarizes the notation used in the paper.

2.1. Continuum Approximation Model. Consider a linear bus route with length $L \mathrm{~km}$. The daily operation time can be divided into $I$ periods, e.g., $I=2$ indicating peak and offpeak hours. For each period $\theta=1, \ldots, I$, the duration time is denoted by $T_{\theta}$ hours. The CA model of bus route design can be expressed as the following minimization problem with the decision variables/functions being headways $h_{\theta}$ and stop density $\delta(x)$ (as a function of location $x$, or equivalently stop spacing function $1 / \delta(x)$ ) (Medina et al. [18]):

$$
\begin{aligned}
\min _{h_{\theta}, \delta(x)} Z= & \sum_{\theta=1}^{I} T_{\theta} \int_{0}^{L}\left(U_{\theta}^{a}(x)+U_{\theta}^{w}(x)+U_{\theta}^{v}(x)\right) \\
& +\left(A_{\theta}^{k}+A_{\theta}^{h}(x)+A_{\theta}^{c}(x)\right) \mathrm{d} x,
\end{aligned}
$$

which is subject to

$$
\begin{aligned}
& \text { vehicle capacity constraint } O_{B}\left(h_{\theta}, \delta(x)\right) \leq K_{B}, \\
& \text { stop capacity constraint } O_{S}\left(h_{\theta}, \delta(x)\right) \leq K_{S}, \\
& \qquad h_{\theta}, \delta(x) \geq 0, \quad \theta=1,2, \ldots, I,
\end{aligned}
$$

where $Z$ is the total generalized system cost, which is the sum of bus users' costs and the agency's costs. The integrands $U_{\theta}^{a}(x), U_{\theta}^{w}(x)$, and $U_{\theta}^{v}(x)$ are patrons' access/egress time cost, waiting time cost, and in-vehicle travel time cost at location $x$ during period $\theta$, respectively. The $A_{\theta}^{k}, A_{\theta}^{h}(x)$, and $A_{\theta}^{c}(x)$ are agency's distance-based cost (irrelevant to location $x$ ), time-based cost, and amortized infrastructure cost during period $\theta$. The $O_{B}\left(h_{\theta}, \delta(x)\right)$ is the maximum vehicle load of the bus route, which is restricted from exceeding vehicle capacity, $K_{B}$ patrons/vehicle, by constraint (1b). Constraint (1c) is the stop capacity constraint that guarantees the maximum amount of waiting patrons $\mathrm{O}_{S}\left(h_{\theta}, \delta(x)\right)$ does not exceed the stop's capacity $K_{S}$ patrons/ stop. Constraint (1d) dictates decision variables/functions being nonnegative. The $U_{\theta}^{a}(x), U_{\theta}^{w}(x), U_{\theta}^{v}(x), A_{\theta}^{k}, A_{\theta}^{h}(x)$, $A_{\theta}^{c}(x), O_{B}\left(h_{\theta}, \delta(x)\right)$, and $O_{S}\left(h_{\theta}, \delta(x)\right)$ can be approximated by $h_{\theta}$ and $\delta(x)$, of which detailed expressions are referred to our previous work [19] and omitted here for the sake of brevity.

From the first-order conditions of (1a)-(1d), the following relationship can be derived for the optimal $h_{\theta}^{*}$ and $\delta(x)^{*}[18,19]$ :

$$
h_{\theta}^{*}=\min \left(\sqrt{f_{\theta}^{h}\left(\delta(x)^{*}, d_{\theta}^{b}(x), d_{\theta}^{a}(x), o b_{\theta}(x)\right)}, h_{\theta}^{\max }\right),
$$

$$
\delta(x)^{*}=\max \left(\sqrt{f_{\theta}^{\delta}\left(h_{\theta}^{*}, d_{\theta}^{\mathrm{b}}(x), d_{\theta}^{\mathrm{a}}(x), o b_{\theta}(x)\right)}, \delta_{\theta}^{\min }\right),
$$

where $h_{\theta}^{*}$ and $\delta(x)^{*}$ are interdependent, i.e., $h_{\theta}^{*} \sim f_{\theta}^{h}\left(\delta(x)^{*}\right)$ and $\delta(x)^{*} \sim f_{\theta}^{\delta}\left(h_{\theta}^{*}\right) ; d_{\theta}^{\mathrm{b}}(x), d_{\theta}^{\mathrm{a}}(x)$, and 
TABle 1: Notation.

\begin{tabular}{|c|c|c|}
\hline Variables & Unit & Descriptions \\
\hline I & & Number of operation time periods \\
\hline$L$ & $\mathrm{~km}$ & Bus route length \\
\hline$N$ & & Number of stops \\
\hline$Z$ & $\mathrm{RMB} /$ day & Total generalized system cost \\
\hline$U_{\theta}^{a}(x)$ & $\mathrm{RMB}$ & Patrons' access/egress time cost at $x$ during period $\theta$ \\
\hline$U_{\theta}^{w}(x)$ & $\mathrm{RMB}$ & Patrons' waiting time cost at $x$ during period $\theta$ \\
\hline$U_{\theta}^{v}(x)$ & $\mathrm{RMB}$ & Patrons' in-vehicle travel time cost at $x$ during period $\theta$ \\
\hline$A_{\theta}^{k}$ & RMB & Agency's distance-based cost during period $\theta$ \\
\hline$A_{\theta}^{h}(x)$ & $\mathrm{RMB}$ & Agency's time-based cost at location $x$ during period $\theta$ \\
\hline$A_{\theta}^{c}(x)$ & $\mathrm{RMB}$ & Agency's amortized infrastructure cost at $x$ during period $\theta$ \\
\hline$U_{\theta}^{a}\left(s_{i}\right)$ & $\mathrm{RMB}$ & Patrons' access/egress time cost during period $\theta$ \\
\hline$U_{\theta}^{v}\left(s_{i}\right)$ & $\mathrm{RMB}$ & Patrons' in-vehicle travel time cost during period $\theta$ \\
\hline$A_{\theta}^{k}\left(s_{i}\right)$ & $\mathrm{RMB}$ & Agency's distance-based cost during period $\theta$ \\
\hline$A_{\theta}^{h}\left(s_{i}\right)$ & RMB & Agency's time-based cost during period $\theta$ \\
\hline$A_{\theta}^{c}\left(s_{i}\right)$ & $\mathrm{RMB}$ & Agency's amortized infrastructure cost during period $\theta$ \\
\hline$T_{\theta}$ & $\mathrm{h}$ & Duration of period $\theta$ \\
\hline$d_{\theta}^{\mathrm{b}}(x), d_{\theta}^{\mathrm{a}}(x)$ & $\mathrm{pax} / \mathrm{km} / \mathrm{h}$ & Boarding and alighting demand density at $x$ during period $\theta$ \\
\hline$p_{i}^{\theta}$ & pax & Onboard flow passing stop $i$ \\
\hline$s_{i}$ & $\mathrm{~km}$ & Location of stop $i$ \\
\hline$S_{k}$ & & The restricted locations to be avoided from being stop locations \\
\hline$l_{i}, r_{i}$ & $\mathrm{~km}$ & Left and right coverage boundaries of stop $i$ \\
\hline$b_{i}^{\theta}, a_{i}^{\theta}$ & $\mathrm{pax} / \mathrm{h}$ & Boarding and alighting volumes at stop $i$ during period $\theta$ \\
\hline & $\mathrm{h}$ & Headway during period $\theta$ \\
\hline$o b_{\theta}(x)$ & $\mathrm{pax} / \mathrm{h}$ & Onboard flow at $x$ during period $\theta$ \\
\hline$\delta(x)$ & Stop $/ \mathrm{km}$ & Stop density at location $x$ \\
\hline$\theta$ & & Subscript representing time periods \\
\hline Parameters & Unit & Descriptions \\
\hline$a_{a}, a_{d}$ & $\mathrm{~m} / \mathrm{s}^{2}$ & Vehicle acceleration and deceleration \\
\hline$K_{B}$ & Pax/vehicle & Vehicle capacity \\
\hline$K_{S}$ & $\mathrm{pax} / \mathrm{stop}$ & Stop capacity \\
\hline$D^{\circ}$ & $\mathrm{km}$ & Minimum distance between bus stops and restricted locations \\
\hline$\gamma_{p}^{a}$ & $\mathrm{RMB} / \mathrm{h}$ & Value of access time \\
\hline$\gamma_{p}^{\text {In }}$ & $\mathrm{RMB} / \mathrm{h}$ & Value of in-vehicle travel time \\
\hline$\gamma_{o}^{\mathrm{cs}}$ & $\mathrm{RMB} / \mathrm{km}$ & Unit cost of distance-based operation cost, e.g., vehicle fuel consumption cost \\
\hline$\gamma_{\mathrm{o}}^{\mathrm{ds}}$ & $\mathrm{RMB} / \mathrm{h}$ & Unit cost of the time-based cost, e.g., drivers' wage and amortized vehicle purchase cost \\
\hline$\gamma^{\mathrm{sc}}$ & $\mathrm{RMB} / \mathrm{h}$ & Unit amortized costs of stop construction \\
\hline$\gamma^{\mathrm{sm}}$ & $\mathrm{RMB} / \mathrm{h}$ & Unit cost of stop maintenance \\
\hline$v_{\mathrm{p}}$ & $\mathrm{km} / \mathrm{h}$ & Patrons' average walking speed \\
\hline$v_{\theta}^{b}$ & $\mathrm{~km} / \mathrm{h}$ & Vehicles' cruising speed during period $\theta$ \\
\hline$t_{\theta}^{l}$ & $\mathrm{~h}$ & Time delay due to bus deceleration and acceleration at stops \\
\hline$t_{b}, t_{a}$ & $\mathrm{~h}$ & Average boarding and alighting delays per passenger \\
\hline & $\mathrm{h}$ & Average delay caused by opening door and closing door \\
\hline
\end{tabular}

$o b_{\theta}(x)$ are demand functions of boarding density, alighting density, and onboard flow at $x$ during period $\theta$, respectively. The $h_{\theta}^{\max }$ is the maximum headway obtained from the vehicle capacity constraint $(1 \mathrm{~b})$. The $\min (\cdot)$ operator in $(2 \mathrm{a})$ guarantees $h_{\theta}^{*}$ no larger than $h_{\theta}^{\max }$, and thus, vehicle load never exceeds the maximum capacity. The $\delta_{\theta}^{\mathrm{min}}$ is the minimum bus stop density obtained from the bus stop capacity constraint (1b). The $\max (\cdot)$ operator in (2b) guarantees $\delta(x)^{*}$ no less than $\delta_{\theta}^{\mathrm{min}}$, and thus, the number of waiting patrons never exceeds the bus stop capacity. The detailed expressions of $f_{\theta}^{h}(\cdot)$ and $f_{\theta}^{\delta}(\cdot)$ can be found in Su et al. and Medina et al. $[18,19]$.

Based on the above analytical results, the efficient algorithm can be readily developed using the iteration method to find the optimal solution (see again in $\mathrm{Su}$ et al. and Medina et al. $[18,19])$. The solution to (1a)-(1d) is, however, still not real design. The $\delta(x)^{*}$ is a continuous function in space, as illustrated in Figure 1, and needs to be discretized into specific stop locations (see the next section for the discretization method).

2.2. Discretization Recipes in Literature. In the literature of CA transit route design models, we found two discretization recipes for translating $\delta(x)^{*}$ into real designs, namely, the "midpoint" and "endpoint" approaches, as demonstrated in Figure 2 (Medina at al. and Wirasinghe and Ghoneim, $[15,18])$. The underlining logic is straightforward: when the integral of the stop density function yields an integer, one stop should be located in the integral interval, e.g., $\left[0, R_{1}\right]$ and $\left[R_{1}, R_{2}\right]$ in Figure 2. Specifically, the midpoint approach locates the stop in the middle of the integral interval, while the endpoint approach locates at the end, as shown in Figure 2. 


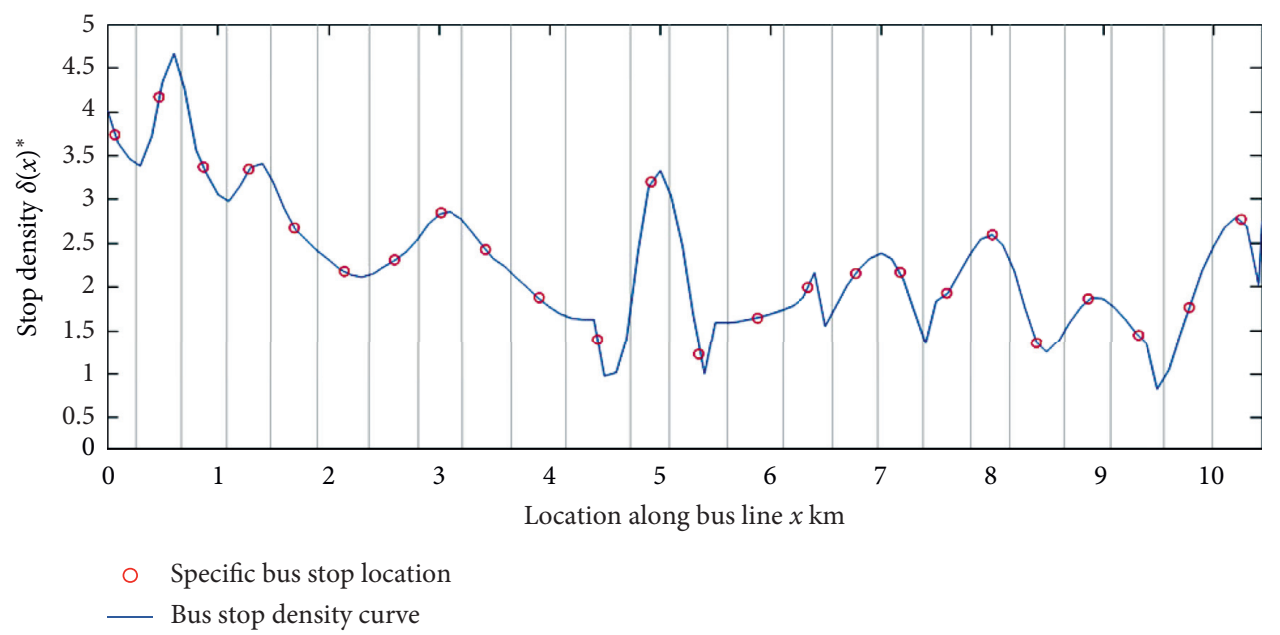

Figure 1: Example of the optimal stop density function $\delta(\mathbf{x})^{*}$ (source: Medina et al. [18]).

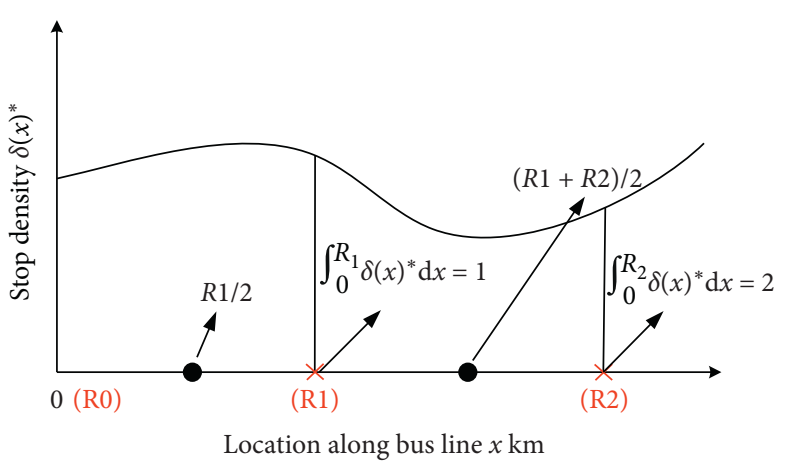

Stop location by using midpoint approach $\times$ Stop location by using endpoint approach

Figure 2: Illustration of two discretization recipes.

The two methods are formulated as follows. First, define $R_{i}\left(R_{0}=0\right)$ as the boundaries that let $\int_{0}^{R_{i}} \delta(x)^{*} \mathrm{~d} x$ yields integer $i=1,2, \ldots, N$, where $N=\int_{0}^{L} \delta(x) \mathrm{d} x$ is the total number of stops along the route. Then, bus stop locations, $s_{i}$, are determined by

$$
\begin{aligned}
& \text { midpoint method } s_{i}=\frac{R_{i-1}+R_{i}}{2}, \quad i=1,2, \ldots, N, \\
& \text { endpoint method } s_{i}=R_{i}, \quad i=1,2, \ldots, N .
\end{aligned}
$$

The endpoint method further includes a default stop at $x=0$, i.e., $s_{0}=0$. Given above stop locations, we obtain the demand coverage of stop $i$ by the left and right boundaries $l_{i}$ and $r_{i}$ :

$$
\begin{gathered}
l_{i}=\left(\frac{s_{i-1}+s_{i}}{2}\right), r_{i}=\left(\frac{s_{i}+s_{i+1}}{2}\right), \\
i=2,3, \ldots, N-1 . \\
l_{1}=0, \\
r_{N}=L .
\end{gathered}
$$

Consequently, discrete system metrics can be computed: e.g., boarding and alighting volumes at each stop by $b_{i}^{\theta}=\int_{l_{i}}^{r_{i}} d_{\theta}^{\mathrm{b}}(x) \mathrm{d} x, a_{i}^{\theta}=\int_{l_{i}}^{P_{i}} d_{\theta}^{\mathrm{a}}(x) \mathrm{d} x$, respectively, and patrons costs and agency costs in the next section.

It is worth noting that although (3) and (4) produce real stop locations, the two discretization recipes have flaws. For instance, they cannot guarantee that the discrete stops are optimally located. This is because both midpoint and endpoint methods neglect the locally nonuniform demand distribution, which apparently impacts the specific locations of stops. In addition, the existing recipes are blinded by ignoring practical location restrictions. The consequence may be improper stop locations that cannot be directly implemented in practice.

2.3. Proposed Discretization Recipe. Other than arbitrarily determining stop locations, we propose a multivariate optimization model to do so and admit constraint of stop locations. Given the knowledge of the total number of stops obtained from the CA model, we accordingly define $N$ variables of stop locations, $s_{i}, i=1,2, \ldots, N$. We also specify the restricted locations to be avoided from being stop locations, $S_{k}, k=1,2, \ldots, K$. Thus, we can formulate the following optimization problem of minimizing the system cost with respect to $s_{i}$ :

$$
\min _{s_{i}} \sum_{\theta=1}^{I} T_{\theta} \sum_{i=1}^{N}\left(U_{\theta}^{a}\left(s_{i}\right)+U_{\theta}^{v}\left(s_{i}\right)\right)+\left(A_{\theta}^{k}\left(s_{i}\right)+A_{\theta}^{h}\left(s_{i}\right)+A_{\theta}^{c}\left(s_{i}\right)\right),
$$

which is subject to

$$
\begin{gathered}
R_{i-1} \leq s_{i} \leq R_{i}, \quad i \in\{1,2, \ldots, N\}, \\
\left|s_{i}-S_{k}\right| \geq D, \quad k \in\{1,2, \ldots, K\}, \\
O_{B}\left(s_{i}\right) \leq K_{B}, \\
O_{S}\left(s_{i}\right) \leq K_{S},
\end{gathered}
$$


where $U_{\theta}^{a}\left(s_{i}\right), U_{\theta}^{v}\left(s_{i}\right), A_{\theta}^{k}\left(s_{i}\right), A_{\theta}^{h}\left(s_{i}\right)$, and $A_{\theta}^{c}\left(s_{i}\right)$ are the corresponding cost items derived based on $s_{i}$. Constraint (6b) defines the feasible space of $s_{i}$. Constraint (6c) restricts stops from being located in the domain of any restricted locations, i.e., $\left[S_{k}-D, S_{k}+D\right], \quad k=1,2, \ldots, K$. Constraints (6d) and (6e) are the capacity constraints of bus vehicles and stops.

Computations of $U_{\theta}^{a}\left(s_{i}\right), U_{\theta}^{v}\left(s_{i}\right), A_{\theta}^{k}\left(s_{i}\right), A_{\theta}^{h}\left(s_{i}\right), A_{\theta}^{c}\left(s_{i}\right)$, and $O_{B}\left(s_{i}\right)$ and $O_{S}\left(s_{i}\right)$ are straightforward, and their expressions are given below:

$$
\begin{aligned}
& U_{\theta}^{a}\left(s_{i}\right)=\gamma_{p}^{a} \int_{l_{i}}^{r}\left(d_{\theta}^{b}(x)+d_{\theta}^{a}(x)\right)\left(\frac{\left|s_{i}-x\right|}{v_{p}}\right) \mathrm{d} x, \\
& i=1,2, \ldots, N, \\
& U_{\theta}^{v}\left(s_{i}\right)=\left\{\begin{aligned}
\gamma_{p}^{\text {in }} p_{i}^{\theta}\left(\frac{s_{i+1}-s_{i}}{v_{\theta}^{b}}+t_{d}\left(s_{i}\right)+t_{\theta}^{l}\right), i=1,2, \ldots, N-1, \\
0, \quad i=N,
\end{aligned}\right.
\end{aligned}
$$

where $p_{i}^{\theta}$ is the onboard flow passing stop $i$, obtained by $p_{i}^{\theta}=\sum_{j=1}^{i} b_{j}^{\theta}-\sum_{j=1}^{i} a_{j}^{\theta}$, and $t_{d}\left(s_{i}\right)=\max \left(t_{b} b_{i}^{\theta}, t_{a} a_{i}^{\theta}\right) h_{\theta}$ is bus dwelling delay at stop $i$ due to passenger boarding and alighting:

$$
\begin{aligned}
& A_{\theta}^{k}\left(s_{i}\right)=\left\{\begin{array}{l}
\frac{\gamma_{o}^{\mathrm{cs}}}{h_{\theta}^{*}}\left(s_{i+1}-s_{i}\right), \quad i=1,2, \ldots, N-1, \\
0, \quad i=N,
\end{array}\right. \\
& A_{\theta}^{h}\left(s_{i}\right)= \begin{cases}\frac{\gamma_{\mathrm{o}}^{\mathrm{ds}}}{h_{\theta}^{*}}\left(\frac{s_{i+1}-s_{i}}{v_{\theta}^{\mathrm{b}}}+t_{d}\left(s_{i}\right)+t_{\theta}^{l}\right), \quad i=1,2, \ldots, N-1, \\
0, \quad i=N,\end{cases} \\
& A_{\theta}^{c}\left(s_{i}\right)= \begin{cases}\gamma^{\mathrm{sc}}+\gamma^{\mathrm{sm}}, \quad i=1,2, \ldots, N-1, \\
0, \quad i=N,\end{cases} \\
& O_{B}\left(s_{i}\right)=\max _{i}\left(p_{i}^{\theta}\right) h_{\theta}^{*}, \\
& O_{S}\left(s_{i}\right)=\max _{i}\left(b_{i}^{\theta}+a_{i}^{\theta}\right) h_{\theta}^{*} .
\end{aligned}
$$

Note in (6a)-(6e) that patrons' waiting cost $U_{\theta}^{w}$ is discarded from the total system cost because it is irrelevant to stop locations. Also note that in (7a)-(7b)-(8a)-(8e), the headways take the optimal $h_{\theta}^{*}$ obtained by the CA model (1a)-(1d).

\section{Solution Method}

Problem (6a)-(6e) is a nonlinear optimization problem with respect to $N$ decision variables, $s_{i}$. The interior-point of the barrier method can be used to solve this problem. For any inequality constraint $\widetilde{f}(x)$ in problem (6a)-(6e), we can use a barrier function $I(x)$ in objective function to replace the inequality constraint $\tilde{f}(x)$. As problem $(6 a)-(6 e)$ is a minimization problem, the used barrier function should produce 0 , when the constraint is satisfied; otherwise, the barrier function produces $\infty$. Therefore, the barrier function can be expressed approximately by

$$
I(x)=-\left(\frac{1}{\xi}\right) \log (-\tilde{f}(x)),
$$

where $\xi$ is a parameter in the approximated barrier function $I(x)$. The larger is the value of $\xi$, the better is the approximated function. Therefore, an iteration process can be used to update the variable of $s_{i}$ by increasing the parameter $\xi$ [20]. In this paper, we directly employ the interior-point algorithm of 'fmincon' function in Matlab 2018a. The "fmincon" function is a built-in program in Matlab to solve the nonlinear problems.

Admittedly, the above solution method does not guarantee a globally-optimal solution due to the nonconvex nature of (6a)-(6e). Thus, we repeated the solutionsearching procedure 10 times for each instance examined in the following numerical studies. Each time, the optimization started with an initial solution generated from the ideal solution to (1a)-(1d) by randomly adjusting stop locations that validate the space constraints (6b) to the neighborhood area. We found that each repetition of the solution procedure always produced the same final solution and thus reckoned that the global optima were attained. Similar treatment can also be found in $\mathrm{Wu}$ et al. and Fan et al. [21, 22].

\section{Numerical Studies}

To demonstrate the effectiveness of the proposed model, Section 4.1 compares two existing discretization recipes via a variety of experiments in a hypothetical bus corridor. Section 4.2 illustrates an application of the proposed model in a case study of a bus route in Chengdu city (China).

4.1. Experimental Comparisons. Following Vaughan and Cousins [23], we consider an arbitrary demand density function as follows:

$$
\lambda(x, y)=\left(\frac{\Lambda}{2}\right)\left[q_{1}(x) q_{2}(y)+q_{2}(x) q_{1}(y)\right],
$$

where $\Lambda$ is the total demand of the corridor; the distributions of trip origins and destinations, $q_{1}(\cdot)$ and $q_{2}(\cdot)$, are assumed to follow a truncated normal distribution, denoted by $\operatorname{Tr} N\left(0, \sigma^{2}, 0, L\right)$ and $\operatorname{Tr} N\left(L, \sigma^{2}, 0, L\right)$ with means of 0 and $L$ $\mathrm{km}$, respectively, and variance of $\sigma^{2}$ being the same. The symmetric setting is purposely made to isolate the findings with regards to the spatial variance of demand. Larger $\sigma$ indicates lower spatial variation, and vice versa. Other parameter values are all retrieved from Li [24]. They are summarized in Table 2.

Experiments are conducted under a variety of demand scenarios with respect to $\sigma \in\{1,2, \ldots, 100\} \mathrm{km}$, $L \in[5,20] \mathrm{km}$, and $\Lambda \in[100,1000] \times L$ passenger $/ \mathrm{h}$. Table 3 summarizes the cost savings of our model as compared to 
TABLE 2: Values of parameters.

\begin{tabular}{lc}
\hline Parameters & Values \\
\hline$v_{p}$ & $1 \mathrm{~m} / \mathrm{s}$ \\
$v_{\text {peak }}^{b}, v_{\text {off-peak }}^{b}$ & $20 \mathrm{~km} / \mathrm{h}, 30 \mathrm{~km} / \mathrm{h}$ \\
$\gamma_{p}^{a}$ & $6.6 \mathrm{RMB} / \mathrm{h}$ \\
$\gamma_{p}^{w}$ & $9.9 \mathrm{RMB} \mathrm{h}$ \\
$\gamma_{p}^{\text {in }}$ & $3.3 \mathrm{RMB} / \mathrm{h}$ \\
$t_{0}^{l}$ & $3 \mathrm{~s}$ \\
$t_{\text {peak }}^{l}, t_{\text {off-peak }}^{l}$ & $5.1 \mathrm{~s} / \mathrm{stop}, 7.64 \mathrm{~s} / \mathrm{stop}$ \\
$a_{a}, a_{d}$ & $1 \mathrm{~m} / \mathrm{s}^{2}, 1.2 \mathrm{~m} / \mathrm{s}^{2}$ \\
$t_{b}$ & $1.55 \mathrm{~s}$ \\
$t_{a}$ & $0.99 \mathrm{~s}$ \\
$\gamma_{o}^{\mathrm{ds}}$ & $37 \mathrm{RMB} / \mathrm{h}$ \\
$\gamma_{o}^{\text {cs }}$ & $2.68 \mathrm{RMB} / \mathrm{km}$ \\
$\gamma^{\text {sc }}$ & $1.67 \mathrm{RMB} / \mathrm{h}$ \\
$\gamma^{\text {sm }}$ & $0.6 \mathrm{RMB} / \mathrm{h}$ \\
$K_{B}$ & $80 \mathrm{pax} / \mathrm{vehicle}$ \\
$K_{S}$ & $120 \mathrm{pax} / \mathrm{stop}$ \\
\hline
\end{tabular}

TABLE 3: Comparisons between the proposed method and existing approaches.

\begin{tabular}{|c|c|c|c|c|c|c|c|}
\hline \multirow{3}{*}{ Parameters } & \multirow{3}{*}{ Cost items } & \multicolumn{6}{|c|}{ Cost saving against, $\%$} \\
\hline & & \multicolumn{3}{|c|}{ Midpoint approach } & \multicolumn{3}{|c|}{ Endpoint approach } \\
\hline & & Max & Min & Avg. & $\operatorname{Max}$ & Min & Avg. \\
\hline \multirow{3}{*}{ Spatial variation, $\sigma^{*}$} & System cost & 0.91 & 0.12 & 0.50 & 2.36 & 0.88 & 1.68 \\
\hline & Passenger cost & 3.36 & -1.43 & 0.27 & -0.76 & -2.68 & -1.82 \\
\hline & Operator cost & 2.18 & -0.28 & 0.61 & 5.39 & 2.56 & 3.41 \\
\hline \multirow{3}{*}{ Corridor length, $L^{* *}$} & System cost & 0.86 & 0.13 & 0.36 & 2.39 & 0.44 & 1.12 \\
\hline & Passenger cost & 2.61 & -0.93 & 0.37 & -0.62 & -5.34 & -1.84 \\
\hline & Operator cost & 1.40 & -0.18 & 0.50 & 6.00 & 2.21 & 3.36 \\
\hline \multirow{3}{*}{ Demand level, $\Lambda^{* * *}$} & System cost & 0.58 & 0.12 & 0.28 & 2.95 & 0.46 & 1.11 \\
\hline & Passenger cost & 1.03 & -2.51 & -0.12 & -0.68 & -3.94 & -1.68 \\
\hline & Operator cost & 2.23 & -0.14 & 0.51 & 6.44 & 1.82 & 3.11 \\
\hline
\end{tabular}

${ }^{*} L$ and $\Lambda$ are set to be $10 \mathrm{~km}$ and $300 \times L$, respectively; ${ }^{* *} \sigma$ and $\Lambda$ are set to be $10 \mathrm{~km}$ and $300 \times L$, respectively; and ${ }^{* * *} \sigma$ and $L$ are set to be $10 \mathrm{~km}$ and $10 \mathrm{~km}$, respectively.

that of midpoint and endpoint methods. It is observed that the proposed method always leads to positive system cost saving in a range of $0.12 \%$ to $2.95 \%$. Comparatively, slightly more savings are found as against the endpoint approach than against the midpoint approach. Closer observation shows that for the midpoint approach, the comparative savings in terms of user cost and agency cost may be negative, but the average savings remain mostly positive. For the endpoint approach, the savings in user cost are always negative, which is opposite for the agency cost. This result can be partly explained by the additional default stop in endpoint design (see Section 2.2), which leads to less user cost but higher agency cost.

Note that the values in Table 3 may look small. This is because the result of the CA model is quite flat at the optimal solution, Estrada et al. [25]. Such benefit will accumulate in the day-to-day operation and become substantial for both bus patrons and agency.

4.2. Case Study in Chengdu. We apply the proposed model to bus route no. 3 in Chengdu (China), as depicted in Figure 3.

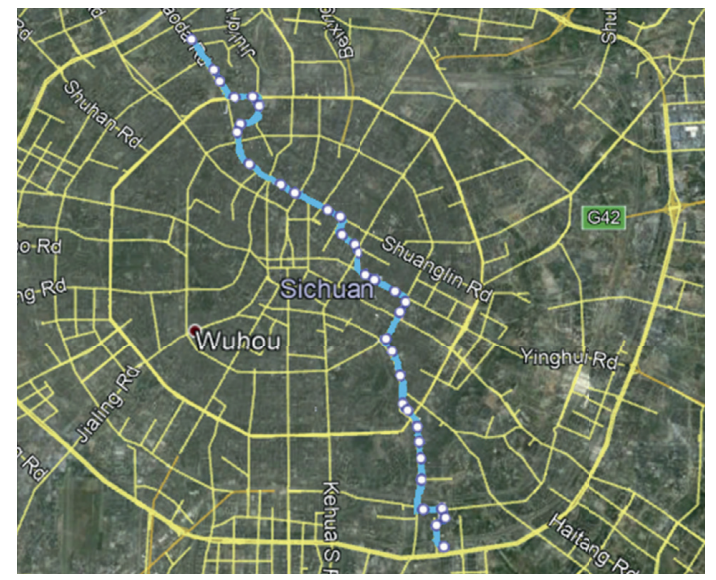

FIGURE 3: The existing bus stop locations of bus route no. 3 in Chengdu (China).

According to our survey, the bus route is of length $18.85 \mathrm{~km}$ and the operation time is between $6: 15 \mathrm{am}$ and $11: 00 \mathrm{pm}$ on weekdays. The peak period is 6 hours on each weekday. Based on historical records, bus vehicles' cruising speed 
TABLE 4: Intersections along bus route no. 3 .

\begin{tabular}{lccc}
\hline Intersection label & Location $(\mathrm{km})$ & Intersection label & Location $(\mathrm{km})$ \\
\hline 1 & 0.17 & 23 & 11.14 \\
2 & 0.5 & 24 & 11.59 \\
3 & 0.78 & 25 & 12.1 \\
4 & 1.08 & 26 & 12.2 \\
5 & 1.71 & 27 & 12.6 \\
6 & 2.42 & 28 & 13.4 \\
7 & 3.01 & 29 & 13.86 \\
8 & 3.58 & 30 & 14.24 \\
9 & 4 & 31 & 14.7 \\
10 & 4.74 & 32 & 15.93 \\
11 & 5 & 33 & 16.04 \\
12 & 5.3 & 34 & 16.29 \\
13 & 6.1 & 35 & 16.59 \\
14 & 6.4 & 36 & 17.28 \\
15 & 6.79 & 37 & 17.61 \\
16 & 7.35 & 38 & 17.86 \\
17 & 7.7 & 39 & 18 \\
18 & 8.6 & 40 & 18.4 \\
21 & 9 & 41 & 18.64 \\
22 & 9.72 & 42 & 18.71 \\
\end{tabular}

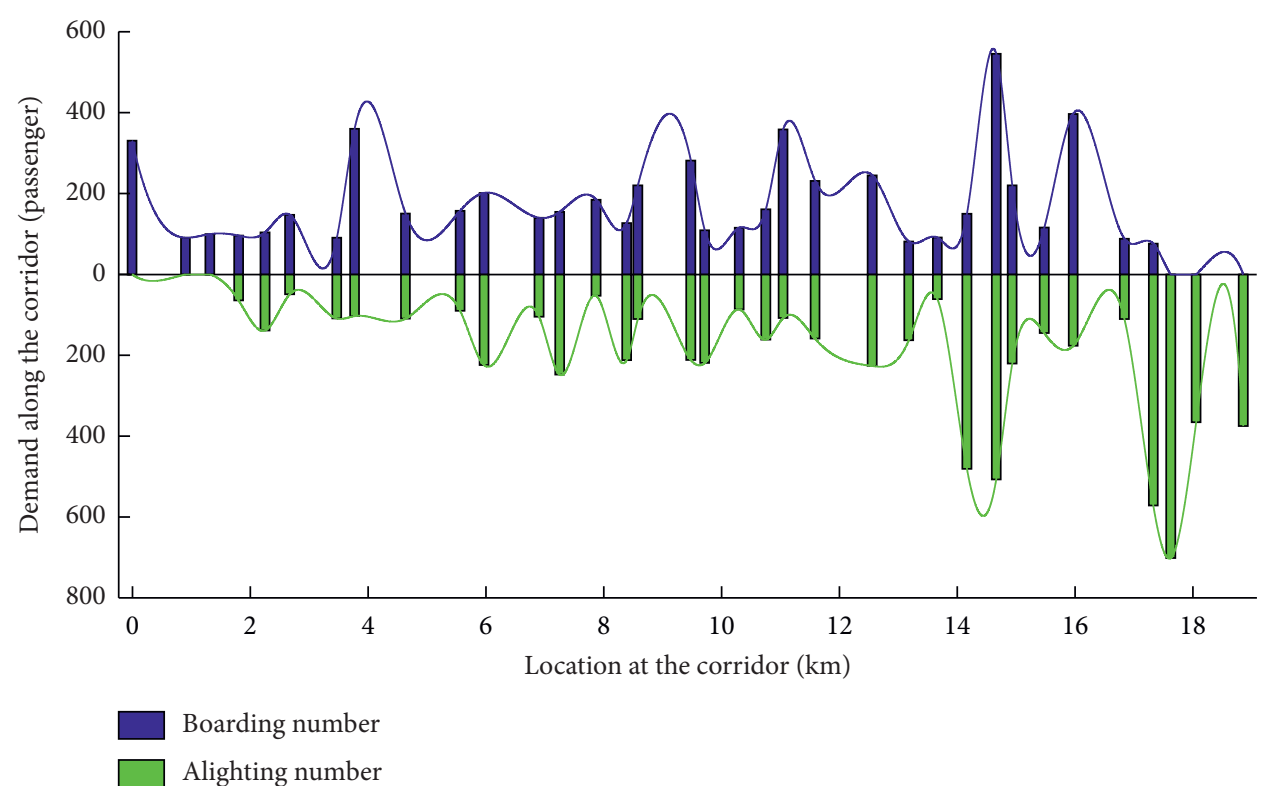

Figure 4: Boarding and alighting demand along the bus route (the peak period, for example).

during peak and off-peak periods are $20 \mathrm{~km} / \mathrm{h}$ and $30 \mathrm{~km} / \mathrm{h}$, respectively. The headways during the peak period and offpeak period are $3 \mathrm{~min}$ and $6 \mathrm{~min}$, respectively. The generation of candidate bus stops follows the policy that the bus stop should keep a minimum distance from restricted locations (e.g., intersections) [26]. Other parameters take the same values in Table 2 as above. Along the bus route contains 43 intersections, whose locations were measured in Google Map and given in Table 4.

In preparation for bus route adjustment, boarding and alighting demand at 35 stops were surveyed on April 10th,
2018. Correspondingly, the density functions of boarding and alighting demand are fitted using spline interpolation, as shown in Figure 4.

Based on the CA model and proposed discretization recipe, we redesign the current transit service. Figure 5(a) presents the optimized bus stop density along the corridor as well as the specific bus stop locations with and without the consideration of location constraint. The stop density ranges from $1.25 \mathrm{stops} / \mathrm{km}$ to $4.42 \mathrm{stops} / \mathrm{km}$. After stop density being discretized, 44 bus stops are determined in the corridor. Figure 5(b) exemplifies two stops, i.e., the 10th and 


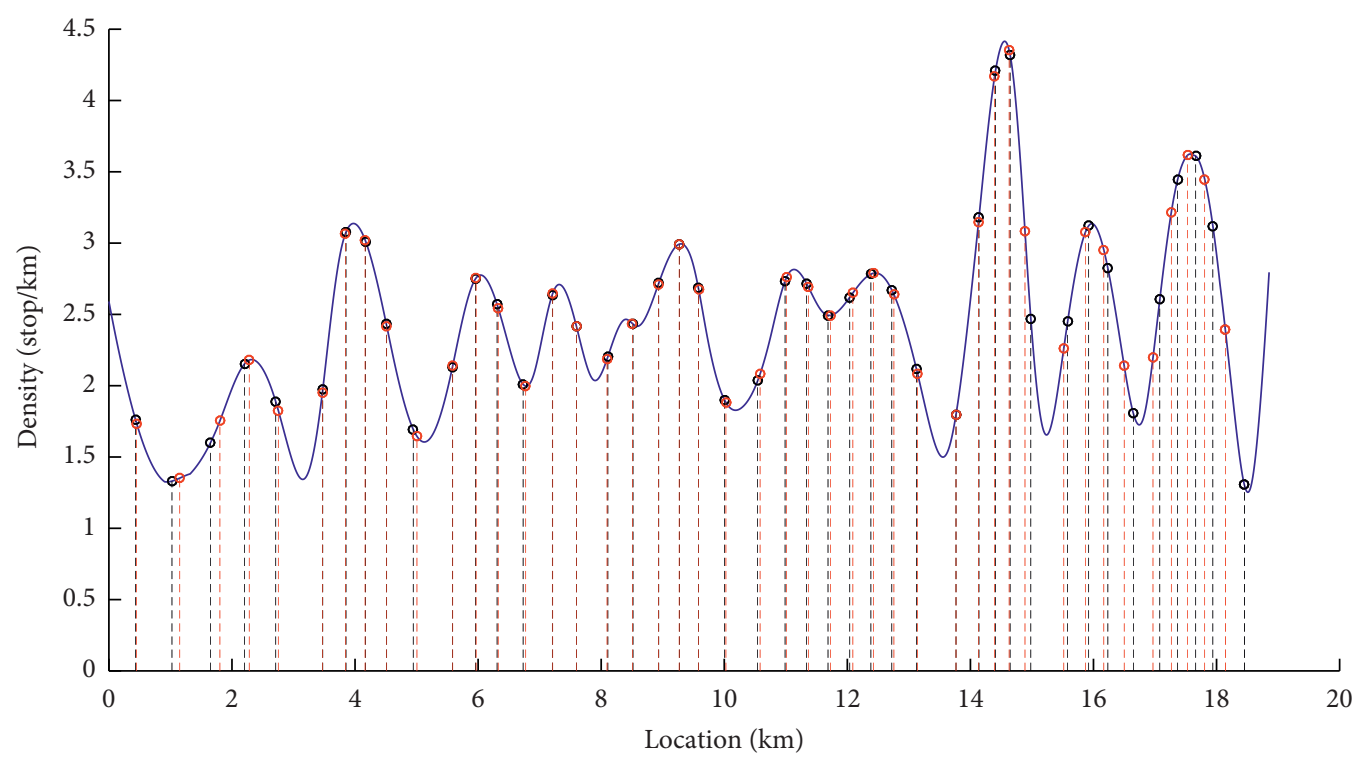

- Optimal bus stop location considering location constraint

- Optimal bus stop location without considering location constraint

— Stop density curve

(a)
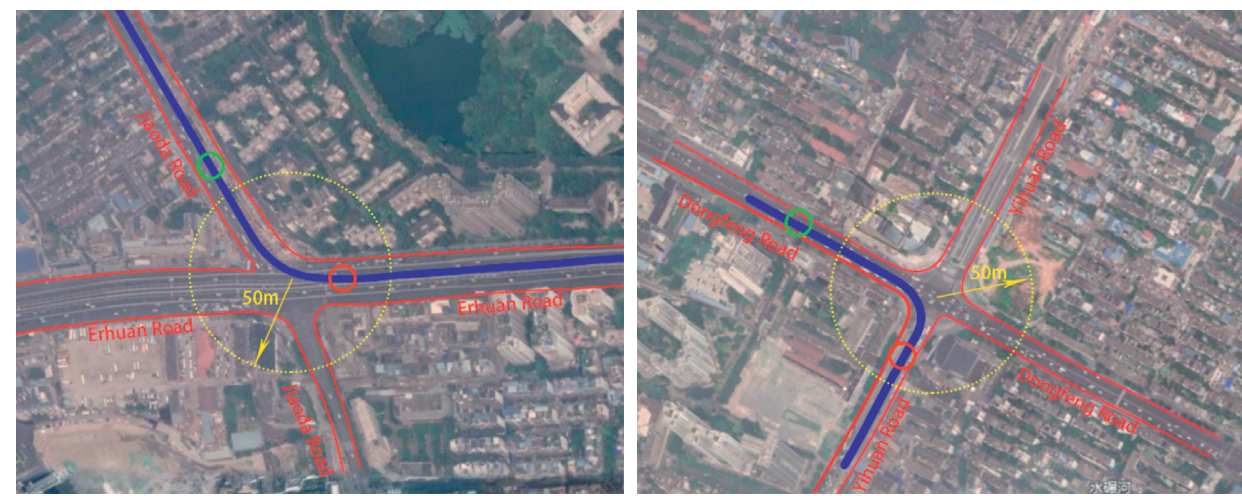

- Optimal design with location constraint

- Optimal design with location constraint

- Idealized design

— Bus line

- Idealized design

_ Bus line

(b)

FIGURE 5: Stop locations for the optimized transit service. (a) Stop locations with and without the location constraint. (b) Locations of 10th and 23th stops for different results.

TABLE 5: Comparison of cost items among different scenarios.

\begin{tabular}{|c|c|c|c|c|}
\hline Scenarios & $\begin{array}{c}\text { Average passenger cost } \\
(\mathrm{RMB})\end{array}$ & $\begin{array}{l}\text { Average agency cost } \\
\text { (RMB) }\end{array}$ & $\begin{array}{c}\text { Average system cost } \\
\text { (RMB) }\end{array}$ & $\begin{array}{c}\text { Location } \\
\text { constraint }\end{array}$ \\
\hline Current service & 5.44 & 5.39 & 10.83 & No \\
\hline Idealized design & 5.68 & 3.42 & 9.10 & Violated \\
\hline $\begin{array}{l}\text { Optimal design with location } \\
\text { constraint }\end{array}$ & 5.66 & 3.46 & 9.12 & Satisfied \\
\hline${ }^{*}$ Difference $(\%)$ & $-0.35 \%$ & $1.65 \%$ & $0.22 \%$ & - \\
\hline
\end{tabular}

*The difference is the calculated by $\left(Z_{I}^{c}-Z_{I}\right) / Z_{I}^{c}, I \in\{p, a, s\}$, where $Z_{p}^{c}, Z_{a}^{c}$, and $Z_{s}^{c}$ are the costs of passenger, agency, and system with the consideration of location constraint, respectively. $Z_{p}, Z_{a}$, and $Z_{s}$ are the costs of passenger, agency, and system without the consideration of location constraint, respectively. 
23th stops. The location constraint is violated by the idealized design while the optimal design with location constraint can guarantee the bus stop location satisfies the location requirement.

Table 5summarizes the current transit service, optimized transit service with and without considering the location constraint. The results show that the optimized result without considering the location constraint is the best but cannot be used into practice due to the constraint violation. Besides, the cost difference between the results with considering and without considering location constraint is very small. The optimized result addressing the location constraint can save $35.81 \%$ agency cost at the expense of $4 \%$ increasing passenger cost, forming $15.79 \%$ system cost saving when compared with the current transit service.

\section{Conclusion}

This paper proposes a modeling framework that connects continuum approximation methods and discrete ones in optimizing bus stop locations. To our best knowledge, this is the first work in the transit route design literature. Our model is no longer limited by the given set of candidate stop locations as the conventional discrete models. Meanwhile, our design outreaches the idealized design of CA models and explicitly addresses practical stop locating restrictions. The proposed hybrid model not only bears the solution efficiency of CA models due to the parsimonious property but also produces implementation-ready designs as do by discrete models. Numerical studies of various scenarios demonstrate the effectiveness of the proposed model. A case study in Chengdu (China) illustrates how the model is applied to bus line redesign/adjustment in reality.

Of note, the present study still has several limitations. For instance, more realistic concerns (e.g., socioeconomic and political ones) are involved in locating bus stops, which may require further fine tuning. The local conditions (e.g., design and safety) of streets may also influence the decision of bus stop location [27]. To account for these constraints, it is expected to develop a decision-support platform based on the proposed modeling framework and integrate other computer aided tools to facilitate designers' operation.

\section{Data Availability}

The boarding and alighting data used to support the findings of this study are available from the corresponding author upon request.

\section{Conflicts of Interest}

The authors declare that they have no conflicts of interest.

\section{Acknowledgments}

This study was funded by the National Nature Science Foundation of China (NSFC 51608455), Sichuan Provincial Science \& Technology Innovation Cooperation Funds
(2020YFH0038), Doctoral Innovation Fund Program of Southwest Jiaotong University (DCX201826), Chongqing Municipal Transportation Engineering Key Laboratory Open Project (2018TE04), and National Key R\&D Program of China (2018YFB1601100).

\section{References}

[1] M.-c. Tan, C. O. Tong, S. C. Wong, and J.-m. Xu, "An algorithm for finding reasonable paths in transit networks," Journal of Advanced Transportation, vol. 41, no. 3, pp. 285305, 2007.

[2] G. Laporte, A. Marín, J. A. Mesa, and F. Perea, "Designing robust rapid transit networks with alternative routes," Journal of Advanced Transportation, vol. 45, no. 1, pp. 54-65, 2011.

[3] Q. K. Wan and H. K. Lo, "A mixed integer formulation for multiple-route transit network design," Journal of Mathematical Modelling and Algorithms, vol. 2, no. 4, pp. 299-308, 2003.

[4] C. Ma and D. Yang, "Public transit network planning in small cites considering safety and convenience," Advances in $\mathrm{Me}$ chanical Engineering, vol. 12, no. 1, Article ID $1687814020902351,2020$.

[5] C. Ma and X. D. Xu, "Providing spatial-temporal priority control strategy for BRT lanes: a simulation approach," Journal of Transportation Engineering, Part A: Systems, vol. 146, no. 7, Article ID 04020060, 2020.

[6] V. R. Vuchic, "Rapid transit interstation spacings for maximum number of passengers," Transportation Science, vol. 3, no. 3, pp. 214-232, 1969.

[7] V. R. Vuchic and G. F. Newell, "Rapid transit interstation spacings for minimum travel time," Transportation Science, vol. 2, no. 4, pp. 303-339, 1968.

[8] J. M. Gleason, "A set covering approach to bus stop location," Omega, vol. 3, no. 5, pp. 605-608, 1975.

[9] A. T. Murray, "A coverage model for improving public transit system accessibility and expanding access," Annals of Operations Research, vol. 123, no. 1-4, pp. 143-156, 2003.

[10] P. G. Furth and A. B. Rahbee, "Optimal bus stop spacing through dynamic programming and geographic modeling," Transportation Research Record: Journal of the Transportation Research Board, vol. 1731, no. 1, pp. 15-22, 2000.

[11] S. I. Chien and Z. Qin, "Optimization of bus stop locations for improving transit accessibility," Transportation Planning and Technology, vol. 27, no. 3, pp. 211-227, 2004.

[12] A. Ceder, M. Butcher, and L. Wang, "Optimization of bus stop placement for routes on uneven topography," Transportation Research Part B: Methodological, vol. 74, pp. 40-61, 2015.

[13] G. F. Newell, "Dispatching policies for a transportation route," Transportation Science, vol. 5, no. 1, pp. 91-105, 1971.

[14] G. F. Newell, "Scheduling, location, transportation, and continuum mechanics: some simple approximations to optimization problems," SIAM Journal on Applied Mathematics, vol. 25, no. 3, pp. 346-360, 1973.

[15] S. C. Wirasinghe and N. S. Ghoneim, "Spacing of bus-stops for many to many travel demand," Transportation Science, vol. 15 , no. 3 , pp. $210-221,1981$.

[16] V. F. Hurdle and S. C. Wirasinghe, "Location of rail stations for many to one travel demand and several feeder modes," Journal of Advanced Transportation, vol. 14, no. 1, pp. 29-45, 1980.

[17] S. C. Wirasinghe and P. N. Seneviratne, "Rail line length in an urban transportation corridor," Transportation Science, vol. 20, no. 4, pp. 237-245, 1986. 
[18] M. Medina, R. Giesen, and J. C. Muñoz, "Model for the optimal location of bus stops and its application to a public transport corridor in Santiago, Chile," Transportation Research Record: Journal of the Transportation Research Board, vol. 2352, no. 1, pp. 84-93, 2013.

[19] Y. Su, X. Liu, G. Lu, and W. Fan, "Optimal design of bus routes for different vehicle types considering various driving regimes and environmental factors," Transportation Research Record: Journal of the Transportation Research Board, vol. 2673, no. 6, pp. 299-316, 2019.

[20] S. Boyd, S. P. Boyd, and L. Vandenberghe, Convex Optimization, Cambridge University Press, Cambridge, UK, 2004.

[21] W. Fan, Y. Mei, and W. Gu, "Optimal design of intersecting bimodal transit networks in a grid city," Transportation Research Part B: Methodological, vol. 111, pp. 203-226, 2018.

[22] L. Wu, W. Gu, W. Fan, and M. J. Cassidy, "Optimal design of transit networks fed by shared bikes," Transportation Research Part B: Methodological, vol. 131, pp. 63-83, 2020.

[23] R. J. Vaughan and E. A. Cousins, "Optimum location of stops on a bus route," in Proceedings of the 7th International Symposium on Transportation and Traffic Theory, Kyoto, Japan, August 1977.

[24] H. Li, "Optimization and case study of transit lines based on continuum approximation," Master's Thesis, Southwest Jiaotong university, Chengdu, China, 2018.

[25] M. Estrada, M. Roca-Riu, H. Badia, F. Robusté, and C. F. Daganzo, "Design and implementation of efficient transit networks: procedure, case study and validity test," Procedia-Social and Behavioral Sciences, vol. 17, pp. 113-135, 2011.

[26] K. Li, Code for Design of Urban Road Public Transportation stop, terminus and Depot Engineering, China Architecture \& Building press, Beijing, China, 2011.

[27] Z. Feng, M. Yang, W. Zhang, Y. Du, and H. Bai, "Effect of longitudinal slope of urban underpass tunnels on drivers' heart rate and speed: a study based on a real vehicle experiment," Tunnelling and Underground Space Technology, vol. 81, pp. 525-533, 2018. 